\title{
Recovery of Soft Tissue Object Deformation from 3D Image Sequences using Biomechanical Models
}

\author{
Xenophon Papademetris ${ }^{1}$, Pengcheng Shi ${ }^{4}$, Donald P. Dione ${ }^{3}$, Albert J. \\ Sinusas $^{23}$, R. Todd Constable ${ }^{2}$, and James S. Duncan ${ }^{12}$ \\ 1 Departments of Electrical Engineering, ${ }^{2}$ Diagnostic Radiology, and \\ 3 Medicine, Yale University New Haven, CT 06520-8042 \\ papad@noodle.med.yale.edu \\ 4 Department of Computer and Information Science, \\ New Jersey Institute of Technology.
}

\begin{abstract}
The estimation of soft tissue deformation from 3D image sequences is an important problem in a number of fields such as diagnosis of heart disease and image guided surgery. In this paper we describe a methodology for using biomechanical material models, within a Bayesian framework which allows for proper modeling of image noise, in order to estimate these deformations. The resulting partial differential equations are discretized and solved using the finite element method. We demonstrate the application of this method to estimating strains from sequences of in-vivo left ventricular MR images, where we incorporate information about the fibrous structure of the ventricle. The deformation estimates obtained exhibit similar patterns with measurements obtained from more invasive techniques, used as a gold standard.
\end{abstract}

\section{Introduction}

There is a class of medical image analysis problems where the goal is the estimation of the displacement field of an object or a group of objects. Examples of such problems are left ventricular (LV) wall motion estimation [9-11] and image guided surgery[4]. In most of these applications, only a relatively sparse set of points, often called landmarks, can be reliably followed on the object from the image data and the estimation of the displacements of remainder of the estimation task can be thought of as interpolation, in other words our problem is: given the displacements of such landmarks, find the best displacements for the rest of the region of interest. Often, however, the displacement estimates of the landmarks are corrupted by noise. In this case, the task becomes an approximation problem, where now the goal is to estimate a displacement field that is close to the originally estimated displacements at the landmark points, and provides reasonable values elsewhere. 


\section{Methods}

We will pose this general problem in a Bayesian-Estimation framework where the goal is to find the displacement field $\hat{u}$ which maximizes the posterior probability:

$$
\hat{u}=\underset{u}{\arg \max } p\left(u \mid u^{m}\right)=\underset{u}{\arg \max }\left(\frac{p\left(u^{m} \mid u\right) \times p(u)}{p\left(u^{m}\right)}\right)
$$

where $u$ is the output displacement field and $u^{m}$ are the original sparse displacement estimates. The prior probability of the measurements $p\left(u^{m}\right)$ is a constant once these measurements have been made and therefore drops out of the minimization process. The first term $p\left(u^{m} \mid u\right)$ will be derived from the noise model assumed in estimating the landmark positions and the second term $p(u)$, the prior probability of the displacement, will be derived from a mechanical model. For a more detailed discussion see[8].

\subsection{Mechanical Model-based priors}

As previously demonstrated by Christiansen et al[2] there is a correspondence between an internal energy function and a Gibbs-Prior. If the mechanical model is described in terms of an internal energy function $W(C, u)$, where $C$ represents the material properties and $u$ the displacement field, then we can write an equivalent prior probability density function $p(u)$ (see equation 1 ) of the Gibbs form:

$$
p(u)=k_{1} \exp (-W(C, u))
$$

We will derive the model term $W$ by a biomechanical model; this can be described in terms of an internal or strain energy function which depends on the deformation of the object and its intrinsic material properties. There are different classes of such models depending on the application; in the case of the left ventricle we will use an anisotropic linear elastic model which will allow us to incorporate information about the preferential stiffness of the tissue along fiber directions[5]. If this method were to be applied to model brain deformation, one could use a model adapted from [6].

Deformation and Strain: Consider a body $B(0)$ which after time $t$ moves and deforms to body $B(t)$. A point $\mathrm{X}$ on $B(0)$ goes to a point $x$ on $B(t)$ and the transformation gradient $F$ is defined as $d x=F d X$. The deformation is expressed in terms of the strain tensor $\epsilon$. Because the deformations to be estimated in this work are bigger than $5 \%$, we use a finite strain formulation, the logarithmic strain $\epsilon^{L}$, which is defined as: $\epsilon=\ln \sqrt{F \cdot F^{\prime}}$. Since the strain tensor is a $3 \times 3$ symmetric 2nd-rank tensor (matrix), we can re-write it in vector form as, $e=$ $\left[\begin{array}{llllll}\epsilon_{11} & \epsilon_{22} & \epsilon_{33} & \epsilon_{12} & \epsilon_{13} & \epsilon_{23}\end{array}\right]^{\prime}$. This will enable us to express the tensor equations in a more familiar matrix notation. 
Strain Energy Function: The mechanical model can be defined in terms of a strain energy function. The simplest useful continuum model in solid mechanics is the linear elastic one which is of the form: $W=e^{\prime} C e$ where $C$ is a $6 \times 6$ matrix and defines the material properties of the deforming body. The left ventricle of the heart is specifically modeled as a transversely elastic material to account for the preferential stiffness in the fiber direction, using the matrix $C$ :

$$
C^{-1}=\left[\begin{array}{cccccc}
\frac{1}{E_{p}} & \frac{-\nu_{p}}{E_{p}} & \frac{-\nu_{f p}}{E_{f}} & 0 & 0 & 0 \\
\frac{-\nu_{p}}{E_{p}} & \frac{1}{E_{p}} & \frac{-\nu_{f p}}{E_{f}} & 0 & 0 & 0 \\
\frac{-\nu_{f p} E_{f}}{E_{p}} & \frac{-\nu_{f p} E_{f}}{E_{p}} & \frac{1}{E_{f}} & 0 & 0 & 0 \\
0 & 0 & 0 & \frac{2\left(1+\nu_{p}\right)}{E_{p}} & 0 & 0 \\
0 & 0 & 0 & 0 & \frac{1}{G_{f}} & 0 \\
0 & 0 & 0 & 0 & 0 & \frac{1}{G_{f}}
\end{array}\right]
$$

where $E_{f}$ is the fiber stiffness, $E_{p}$ is cross-fiber stiffness and $\nu_{f p}, \nu_{p}$ are the corresponding Poisson's ratios and $G_{f}$ is the shear modulus across fibers. $\left(G_{f} \approx\right.$ $E_{f} /\left(2\left(1+\nu_{f p}\right)\right)$. If $E_{f}=E_{p}$ and $\nu_{p}=\nu_{f p}$ this model reduces to the more common isotropic linear elastic model. Alternatively a different form of $W$ altogether could be used such as the one from a Rivlin-Mooney Material Model[6].

\subsection{Landmark displacement estimation}

In our work, the original displacements on the outer surfaces of the myocardium were obtained by using the shape-tracking algorithm whose details where presented in [11]. We note that other displacement data, including that from magnetic resonance tagging $[9,10]$, could also be used.

The shape-tracking algorithm also produces a set of confidence measures for each match. We model these estimates with a Gaussian noise model and generate the term $p\left(u^{m} \mid u\right)$ of equation (1) to be

$$
p\left(u^{m} \mid u\right)=\frac{1}{\sqrt{2 \pi \sigma^{2}}} e^{\frac{\left(u-u^{m}\right)^{2}}{2 \sigma^{2}}}
$$

where $\sigma^{2}$ is set to be the reciprocal of the confidence of the particular displacement estimate. Where no displacements estimates are available the confidence is set to zero.

\subsection{Solution using the Finite Element Method}

Having defined both the model $p(u)$ and data $p\left(u^{m} \mid u\right)$ portions of the problem, we can now minimize equation (1) to find the optimal displacement field $\hat{u}$. Taking logarithms and differentiating with respect to the displacement field $u$ results in a system of partial differential equations, which we solve using the Finite Element Method[1]. The first step in the finite element method is the division or tessellation of the body of interest into elements; these are commonly tetrahedral or hexahedral in shape. Once this is done, the partial differential 
equations are written down in integral form for each element, and then the integral of these equations over all the elements is taken to produce the final set of equations. For more information one is referred to standard textbooks such as Bathe[1]. The final set of equations is then solved to produce the output set of displacements.

\section{$3 \quad$ Results}

In this section we present results from the application of this methodology to ten sets of cardiac MR sequences acquired from anesthetized dogs. The resulting 3D image set consists of sixteen 2D image slices per temporal frame, and sixteen temporal 3D frames per cardiac cycle. First the dogs were positioned in the magnet for initial imaging under baseline conditions. The left anterior descending coronary artery was then occluded and a second set of images was acquired. The images were pre-segmented to extract the endo- and epi-cardial boundaries surfaces and interactively corrected using a platform specially developed for this purpose[7]. Then points on the corresponding surfaces were tracked to generate the input displacement data using shape-based algorithms described in [11]. The myocardium was modeled as an anisotropic linear elastic material which was stiffer in the fiber directions[5]; shown in figure 1. The tissue was assumed to be 3.3 times stiffer along the fiber direction, obtained by linearization of the non-linear model from [5], and approximately incompressible.

For each frame between end-systole (ES) and end-diastole (ED), a two step problem is posed: (i) solving equation (1) normally and (ii) adjusting the position of all points on the endo-and epi-cardial surfaces so they lie on the endo- and epicardial surfaces at the next frame using a modified nearest-neighbor technique and solving equation (1) once more using this added constraint. This ensures that there is no bias in the estimation of the radial strain. Figure 2 shows a contour map of radial strain (thickening) in a long-axis section of a normal left ventricle and in the same animal after occlusion.

\begin{tabular}{|l|c|c|c|c|}
\hline Percentage change & Radial normal & Radial Infarct & Circum. Normal & Circum. Infact \\
\hline Our Method (Average) & $-16.4 \%$ & $-135.1 \%$ & $+18.9 \%$ & $+77.2 \%$ \\
Sonomicroemeters[3] & $+5.6 \%$ & $-150.0 \%$ & $+15.4 \%$ & $+73.3 \%$ \\
\hline
\end{tabular}

Table: Radial and Circumferential Percentage Strain Changes for Normal and Infarcted Regions.

The validation measures used were the percentage end-systolic strain change for the radial and circumferential components between the baseline and postocclusion measurements. The normal and infarcted regions where defined by post-mortem measurements. These results are compared to measurements made by using implanted sonomicrometers, work performed by members of our research team and reported in[3], which provide highly accurate strain measurements by calculating relative Doppler-based displacements, and are used as a 


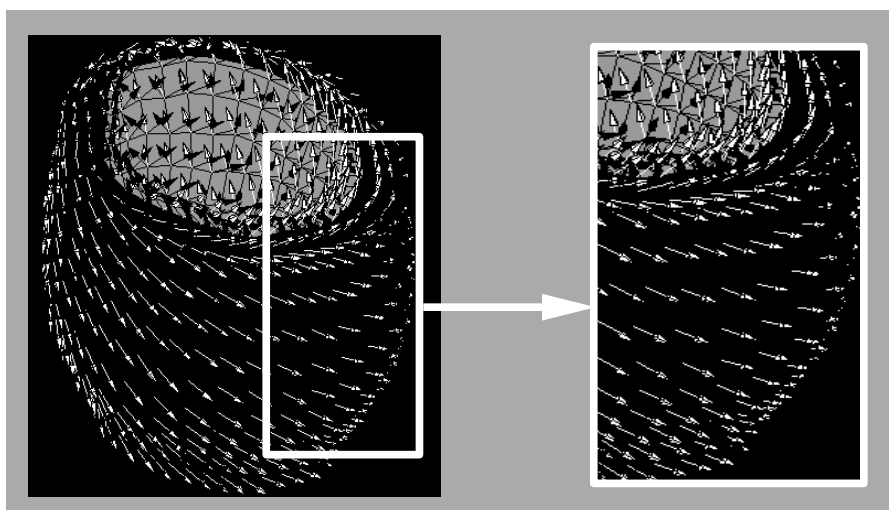

Fig. 1. Fiber direction in the left ventricle as defined in Guccione et al [5].

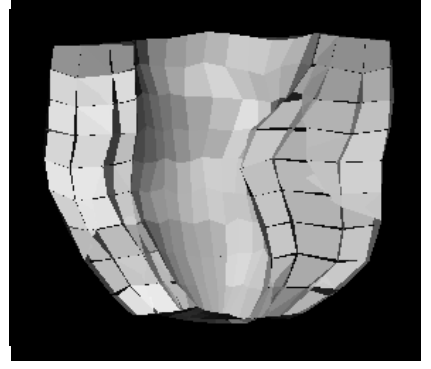

Base-line Study

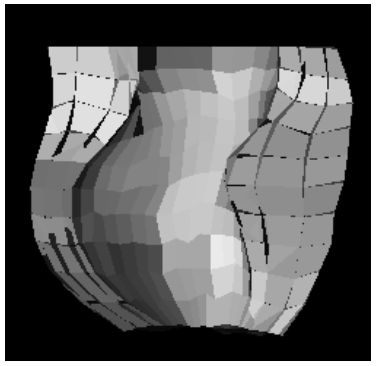

Post-Infarct Study

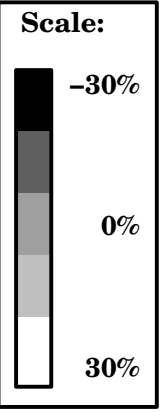

Fig. 2. Radial Strain at end-systole in a section normal left-ventricle (left) and postocclusion(right) shown in an a long-axis sectional view. Normal behavior is thickening (positive). Note the infarct region on the right which is in darker color.

gold standard. The results are summarized in the table and are consistent with the observation that in the case of infarction the tissue thins instead of thickens, hence there is a negative change in the radial strain and it bulges out instead of contracting, explaining the positive change in the circumferential strain. For a more detailed discussion see a related technical report[8].

\section{Conclusions}

In this paper we have described a methodology for the estimation of deformation from sequences of 3D images of individual objects, using the left ventricle of the heart as a key example. We believe that the best approach to this problem involves the modeling of the mechanical properties of the object explicitly in the language of continuum mechanics, as this makes possible the incorporation of existing theoretical and experimental research in biomechanics, and it provides a growth path for solving more difficult problems by naturally invoking more sophisticated/appropriate models. In this cardiac work for example, we were 
able to easily take advantage of knowledge of fiber orientation to create a model of the heart that is anisotropic and accounts for more of the actual properties of the tissue. In the future, we hope to use a non-linear mechanical model which will capture the 'hardening' of the tissue as it is stretched. We also note that the only part of this work that is specific to the left ventricle is the particular strainenergy function. By substituting an appropriate matrix $C$ in the case of a linear elastic material or an altogether different form of $W$ in equation (2) altogether, this method can be used to estimate the deformation of other objects.

\section{Acknowledgments}

The first author would also like to thank professors Turan Onat and Gary Povirk from the Department of Mechanical Engineering at Yale University for their help.

\section{References}

1. Bathe. K. Finite Element Procedures in Engineering Analysis. Prentice-Hall, New Jersey, 1982.

2. Christiansen G. E., Rabbitt R. D., and Miller M. I. 3D Brain mapping using deformable neuroanatomy. Physics in Medicine and Biology, 39:609-618, 1994.

3. Dione D. P., Shi P., Smith W, De Man P., Soares J., Duncan J.S., and Sinusas A.J. Three-dimensional regional left ventricular deformation from digital sonomicrometry. In 19th Ann. Int. Conf. of the IEEE Engineering in Medicine and Biology Society, pages 848-851, Chigago, IL, March 1997.

4. Edwards P. J., Hill D.L.G., Little J.A., and Hawkes D.J. Deformation for image guided interventions using a three component tissue model. In Information Processing in Medical Imaging, pages 218-231, Vermont, USA, June 1997.

5. Guccione J. M. and McCulloch A. D.. Finite element modeling of ventricular mechanics. In Hunter P.J., McCulloch A. D., and Nielsen P., editors, Theory of Heart, pages 122-144. Springer-Verlag, Berlin, 1991.

6. Mendis K.K., Stalnaker R.L., and Advani S.H. A constitutive relationship for large deformation finite element modeling of brain tissue. Journal of Biomechanical Engineering, 117(3):279-85, 1995.

7. Papademetris X., Rambo J., Dione D.P, Sinusas A.J., and Duncan J.S. Visually interactive cine-3D segmentation of cardiac mr images. Suppl. Journ. of the American College of Cardiology Volume 31, \#2 (Supplement A), February 1998.

8. Papademetris X. and Shi P. and Dione D.P. and Sinusas A.J. and Constable R.T. and Duncan J.S. Recovery of Soft Tissue Object Deformation from 3D Image Sequences using Biomechanical Models Technical Report 1999-01, Image Processing and Analysis Group, Dept. of Diagnostic Radiology, Yale University, March 1998.

9. Park J., Metaxas D., and Axel L.. Volumetric deformable models with parameter functions: a new approach to the 3D motion analysis of the LV from MRI-SPAMM. In Fifth International Conference on Computer Vision, pages 700-705, 1995.

10. Prince J. L. and McVeigh E. R. Motion estimation from tagged mr image sequences. IEEE Transactions on Medical Imaging, 11:238-249, June 1992.

11. Shi P., Sinusas A.J., Constable R.T., Ritman E., and Duncan J.S. Point-tracked quantitative analysis of left ventricular motion from $3 \mathrm{D}$ image sequences. IEEE Transactions on Medical Imaging, in-press. 